\title{
Percepciones de los directivos de los programas de formación de ginecólogos y obstetras sobre las políticas de aborto en hospitales académicos de los EE.UU.
}

\author{
Perceptions of directors of gynecology and obstetrics training programs on abortion policies in US academic hospitals
}

Comentado de:

Zeldovich VB, et al. Obstet Gynecol 2020; 135:1296-305. PMID: $32459421^{1}$

\section{Objetivo}

Evaluar la prevalencia y las características de las políticas que regulan el aborto en los hospitales académicos de los EE.UU.

\section{Diseño}

Estudio de corte transversal, de tipo mixto (encuesta y entrevistas cualitativas).

\section{Lugar}

Hospitales escuela de las cuatro regiones de los EE.UU. con residencias de Ginecología y Obstetricia con programas acreditados por el Consejo de Acreditación de Educación Médica para Graduados (ACGME, por sus iniciales en inglés) eligiendo en forma selectiva una muestra del Programa de Capacitación de Residencia Kenneth J. Ryan en Aborto y Planificación Familiar (asociado a un compromiso explícito con la capacitación sobre el aborto). Fueron incluidas algunas instituciones con afiliación católica. Fueron excluidos los programas militares y los de Puerto Rico.

\section{Participantes}

Fueron invitados a participar los directores de los centros de formación por considerarse las personas más conocedoras de las políticas y prácticas de aborto en cada lugar. Participaron 13 directores de diez sitios diferentes en la primer entrevista (2014), y cinco directores de cinco lugares diferentes en la segunda (2017), excluyendo los hospitales que pertenecían al programa Ryan.

El Programa de Capacitación de Residencia Kenneth J. Ryan en Aborto y Planificación Familiar es un programa nacional coordinado desde el Centro Bixby de la Universidad de California, San Francisco. Fue fundado en 1999 con el objetivo de integrar y mejorar formalmente la capacitación en planificación familiar para residentes en obstetricia y ginecología a través de servicios ambulatorios de interrupción del embarazo en hospitales universitarios o mediante socios comunitarios de colaboración.

La encuesta nacional (2015) fue realizada mediante un cuestionario autoadministrado, enviado por correo a 231 directores.

\section{Métodos}

Fueron realizadas dos entrevistas semiestructuradas y una encuesta nacional autoadministrada. Las entrevistas constaban de preguntas abiertas, llevadas a cabo por un sociólogo especialista en la temática. En la primera (2014) fueron exploradas las percepciones sobre las políticas de aborto hospitalario y los procesos de aprobación. La segunda entrevista (2017), al cierre de la encuesta nacional, fue realizada con el objetivo de equilibrar la muestra por tipo de residencia y lograr una saturación temática suficiente. La gestión de los datos de ambas instancias se realizó mediante el software Atlas.ti y el análisis, mediante el enfoque de la teoría fundamentada.

La encuesta nacional (2015 a 2016) fue diseñada sobe la base de la primera entrevista. El cuestionario autoadministrado fue enviado por correo electrónico con algunas preguntas de opción múltiple y otras de respuesta abierta. Las preguntas estaban orientadas a conocer la prevalencia de diferentes políticas y los distintos mecanismos de aplicación. Se analizó la tasa de respuesta por región mediante una serie de modelos de regresión logística.

\section{Resultados}

La tasa de respuesta de la encuesta fue de $73 \%$. De 169 participantes en total, ocho tenían afiliación católica; 53 pertenecían al noreste de los EE.UU., 35 al medio oeste, 54 al sur y 27 al oeste. El tiempo de permanencia en los puestos de trabajo era 12,5 +/- 9,5 años. Los sitios sin afiliación católica tuvieron una tasa de respuesta que los de afiliación católica ( 76 vs $42 \%$; $p<0,01$ ). El $57 \%$ de los encuestados informó que su hospital tenía algún tipo de política que restringía el aborto, más allá de las leyes de su estado.

Para el análisis de las políticas se definieron cinco aspectos principales:

\section{Políticas según motivo dado para el aborto}

Los encuestados dividieron las razones en "no indicados médicamente o electivosz "médicamente indicados". Se registró que $48 \%$ de los sitios restringían los abortos electivos y $28 \%$, los médicamente indicados. Respecto a las restricciones de los abortos electivos, a nivel regional se observó una mayor proporción en el medio oeste (63\%) y el sur (61\%), que podían estar explicadas por diferencias en las edades gestacionales. En el caso de los abortos médicamente indicados, fueron registrados una amplia variedad de motivos, entre los que se destacaron las indicaciones maternas y fetales como las más utilizadas para justificar el aborto. La salud mental materna, la violación o el incesto fueron las más restringidas por las políticas hospitalarias.

\section{Formato de las políticas}

Frente a la limitación del aborto, $31 \%$ de los hospitales tenían políticas escritas y $33 \%$, no escritas. Fue más probable que las restricciones sobre el aborto electivo estuvieran escritas (30\%) que las restricciones sobre los abortos indicados médicamente (15\%). El análisis cualitativo sugiere que este tipo de política "no escrita"genera confusión, y también, mayor comodidad para los profesionales al permitirles ejercer una practica más flexible.

\section{Mecanismo de aplicación de las políticas}

El $57 \%$ de los sitios requería la aprobación y el $34 \%$ requería la notificación de alguien para realizar un aborto, siendo los especialistas en medicina materno-fetal (53\%), el comité de ética hospitalaria (45\%) y los jefes de departamento de obstetricia y ginecología (43\%) las instancias de aprobación más solicitadas. Por otro lado, quienes más comúnmente solicitaron notificación fueron los jefes de los departamentos de obstetricia y ginecología $(40 \%)$, los especialistas en medicina materno-fetal (33\%) y el comité de ética hospitalaria o de aborto (14\%).

\section{Fuente de las políticas}

Aquellas escritas fueron establecidas con mayor frecuencia por los ejecutivos del hospital ( $57 \%$ ), los jefes de departamentos de 
obstetricia y ginecología (51\%) y los abogados (45\%). En cambio, las políticas no escritas fueron determinadas por los jefes de los departamentos de obstetricia y ginecología (49\%), las enfermeras y la administración de enfermería (45\%), los especialistas en medicina materno-fetal y otros médicos, obstetras y ginecólogos, desempeñando papeles importantes (ambos con 38\%).

\section{Razones de las fuentes para restringir el aborto}

Las razones identificadas con mayor frecuencia fueron el deseo de evitar controversias que pudieran tener repercusión sobre los medios de comunicación, las creencias personales, el deseo de evitar a manifestaciones en contra y la preocupación por la pérdida de financiamiento. Algunos identificaron además a la presión de los políticos y a la seguridad laboral personal. Cabe mencionar que las respuestas fueron simila- res tanto para las políticas escritas como para las no escritas.

Conflicto de interés de los autores: Callie Langton reveló que es empleada en la fundación Susan Thompson Buffett. Los otros autores no reportaron potenciales conflictos de interés.

\section{Conclusiones}

Las políticas institucionales reflejaron restricciones al aborto, más allá de las previstas por las leyes estatales, que respondían a intereses institucionales amplios y funcionaban en detrimento del acceso de los pacientes a la práctica y de las oportunidades de capacitación médica. Las políticas de aborto vagas o no escritas dieron a los proveedores de atención médica algo de flexibilidad sobre sus prácticas.

\section{Comentario}

El aborto es una práctica sanitaria de alta prevalencia en el mundo. Este estudio presenta datos cualitativos que permitieron evidenciar los matices de las políticas, que a veces eran complejas o confusas. Tanto los encuestados como los entrevistados informaron que las políticas de aborto hospitalario perpetuaban un sistema de juicios de valor sobre las razones de las pacientes para solicitar un aborto. Esta estratificación de la legitimidad de la práctica sirve para reforzar el estigma del aborto, tanto para los pacientes como para los médicos en formación. Las políticas no escritas dieron a los proveedores de atención médica más flexibilidad, especialmente en casos complicados donde las pacientes no cumplían con los criterios para el aborto en sus hospitales. Aunque el objetivo teórico de los mecanismos de aprobación y ejecución era brindar objetividad y apoyo a una decisión clínica difícil, los entrevistados percibieron estas estrategias como medidas de protección del hospital de políticas y riesgo financiero en lugar cumplir una función médica, legal o ético.

Entre las fortalezas del estudio destacamos su alta tasa de respuesta de la encuesta ( $73 \%$ ) y la importancia de los datos cualitativos frente al análisis de una temática compleja mediante las entrevistas. Entre las limitaciones de los datos cualitativos, se evidencia una representación excesiva de sitios con programas de capacitación Ryan y una insuficiente representación de las instituciones religiosas y del medio oeste, lo que puede resultar en una subestimación de las restricciones a la práctica del aborto. Además es posible que los resultados no sean generalizables a hospitales no docentes de los EE.UU.

En Argentina, el 29 de Diciembre de 2020 el Congreso Nacional sancionó la Ley Nro. 27.610 que tiene como objeto regular el acceso a la interrupción voluntaria del embarazo (IVE) y la atención postaborto ${ }^{2,3}$. Sin embargo, aún con la existencia de esta ley, la falta de políticas institucionales claras, podría ocasionar problemas al momento de garantizar el derecho a la IVE. Entendemos la preocupación a la que se enfrenta el sistema de salud ante la problemática del aborto y la necesidad de prestar una atención adecuada basada en políticas claras y escritas, que respeten los derechos de las pacientes y acompañen la formación del personal de salud. Por este motivo, a mediados de 2020, en contexto del creciente debate en los años previos a la sanción de la ley 27.610, mientras aún se encontraba vigente la legislación que regulaba la interrupción del embarazo bajo determinados causales ${ }^{4}$, para explorar la nuestra experiencia institucional y enriquecer la discusión de los hallazgos del estudio resumido en este artículo, se realizó una encuesta a médicos del Servicio de Medicina Familiar del Hospital Italiano de Buenos Aires. De 41 respuestas, $83 \%$ de los participantes declaró que tenía conocimiento sobre la legislación vigente que en ese momento regulaba la practica del aborto en la Ciudad Autónoma de Buenos Aires (CABA). Destacamos que $17 \%$ refirió que no le quedaba clara esta legislación. De estos, más de la mitad manifestó que sabía donde encontrarla si necesitaba consultarla. Por otro lado, manifestaron que las políticas institucionales eran diferentes a las estatales el $58,5 \%$ de los encuestados, mientras que $39 \%$ de los participantes consideraron que existían políticas institucionales que restringen el aborto por fuera de la ley, percibiéndose por el $61,5 \%$ como no escritas y poco claras. Este carácter poco claro de las políticas representaba, para el $92,7 \%$ de los participantes, falta de respaldo en su práctica e incomodidad al decidir por su voluntad. Entre las razones consideradas como posible fundamento de las políticas restrictivas se mencionaron en primer lugar el deseo de evitar la controversia, la presión política y el desconocimiento del alcance legal, y $87,5 \%$ de los participantes creyeron que las dichas políticas pueden consistir en un obstáculo para la formación médica.

Cabe destacar que, a mediados de 2020, a partir de algunos casos de solicitud de interrupción legal del embarado recibidas por el Hospital Italiano de Buenos Aires, la Dirección Médica de esta institución solicitó a un grupo de profesionales de diversos servicios, el desarrollo de una normativa institucional que especifique la manera en que todos los profesionales de la salud debían dar respuesta a esta práctica. En coincidencia, en el mes de Julio de 2020, la legislatura de la CABA dictaminó la adhesión de la Ciudad al protocolo Nacional para la Atención Integral de las personas con derecho a la Interrupción Legal del Embarazo, normativa que involucra también al subsector de la seguridad social y privado.

\section{Conclusiones de las comentadoras}

En Argentina, aún con la sanción de la ley de interrupción voluntaria del embarazo a fines de 2020, la legitimidad del aborto sigue estando en foco de discusión continua. Comprender la naturaleza, la etiología y los árbitros de las políticas que restringen dicha práctica es el primer paso para preservar el aborto institucionalizado como un recurso vital tanto para pacientes, como para los médicos en formación. El análisis del trabajo resumido permite destacar la necesidad imperiosa de que las 
políticas hospitalarias que se desarrollen en torno a la práctica médica, se expliciten en forma clara y escrita, y que se rijan según las dictaminadas por las leyes estatales. Este es un paso clave para una mayor homogeneidad en la prestación de servicios que contemplen los derechos de las pacientes y para que las prácticas de los médicos en formación puedan ser luego reproducidas en otros ámbitos y coincidan con las necesidades poblacionales actuales.

Natalia Ponce de Leon, Agustina Vega, Daniela Epstein [ Servicio de Medicina Familiar y Comunitaria, Hospital Italiano de Buenos Aires. natalia. poncedeleon@hospitalitaliano.org.ar, agustina.vega@hospitalitaliano.org.ar, daniela.epstein@hospitalitaliano.org.ar ]

Ponce de Leon N, Vega A, Epstein D. Percepciones de los directivos de los programas de formación de ginecólogos y obstetras sobre las políticas de aborto en hospitales académicos de los EE.UU. . Evid Actual Pract Ambul. 2021;24(1):e002122. Comentado de: Zeldovich VB, et al. Abortion Policies in U.S. Teaching Hospitals: Formal and Informal Parameters Beyond the Law. Obstet Gynecol. 2020;135(6):1296-1305. PMID: 32459421

\section{Referencias}

1. Zeldovich VB, Rocca CH, Langton C, et al. Abortion Policies in U.S. Teaching Hospitals: Formal and Informal Parameters Beyond the Law. Obstet Gynecol. 2020;135(6):1296-1305. Available from: 10.1097/AOG.0000000000003876.

2. Argentina. Ministerio de Justicia y Derechos Humanos. Presidencia de la Nación. Ley 27.610. Acceso a la interrupción voluntaria del embarazo. ; 2021. Available from: https://www.boletinoficial.gob.ar/detalleAviso/primera/239807/20210115.

3. Discacciati V, Epstein D, Musarella N, et al. En 2020, el aborto en Argentina salió de la clandestinidad. Evid Actual Pract Ambul. 2021;24(1):21152115. Available from: http://www.evidencia.org.ar/index.php/Evidencia/article/view/6910\#R97933021183792.

4. Discacciati V. En Argentina, durante 2018 el aborto salió del armario pero no de la clandestinidad. Evid Actual Pract Ambul. 2018;21(2):42-44. Available from: http://www.evidencia.org.ar/index.php/Evidencia/article/view/6804. 